\title{
Review
}

\section{Cognitive Behaviour Therapy for Depression and Anxiety in Parkinson's Disease}

\author{
Sarah J. Egan ${ }^{\mathrm{a}, *}$, Ken Laidlaw ${ }^{\mathrm{b}}$ and Sergio Starkstein ${ }^{\mathrm{c}}$ \\ ${ }^{\mathrm{a} S}$ School of Psychology and Speech Pathology, Curtin University, WA, Australia \\ ${ }^{\mathrm{b}}$ Department of Clinical Psychology, The University of East Anglia, Norfolk, UK \\ ${ }^{\mathrm{c}}$ School of Psychiatry, University of Western Australia, Crawley WA, Australia
}

\begin{abstract}
Evidence is reviewed demonstrating that cognitive behavior therapy (CBT) is effective in the treatment of depression and anxiety in Parkinson's disease. The aims were to review the extant literature, specify a model of cognitive and behavioral maintenance factors in depression and anxiety in Parkinson's disease and provide a guide to treatment. It is argued that treatment should take into account specific cognitive and behavioral maintaining factors. Symptoms of depression and anxiety are highly prevalent in Parkinson's disease and therapists should consider how to augment the efficacy of CBT for patients with Parkinson's disease. Cognitive and behavioral interventions can help people overcome some of the challenges in living with PD by maximizing wellbeing and overall quality of life.
\end{abstract}

Keywords: Parkinson's disease, anxiety, depression, model, CBT

Parkinson's Disease (PD) is a common, chronic and progressive neurological condition associated with negative impact on quality of life, disability and increased mortality $[1,2]$. We have recently outlined a rationale for considering the use of cognitive behavior therapy (CBT) for treatment of depression and anxiety in Parkinson's disease [3]. The current paper adds a new element to the literature by proposing a conceptual framework for applying CBT for depression and anxiety in PD. The paper also addresses strategies for overcoming barriers to treatment engagement in PD.

\section{PREVALENCE AND IMPACT OF ANXIETY AND DEPRESSION IN PARKINSON'S DISEASE}

Depression in PD is very common, with prevalence rates ranging from 50 per cent [4] to 75 per cent [5]. PD patients with depression have faster cognitive

\footnotetext{
*Correspondence to: Sarah Egan, School of Psychology and Speech Pathology, Curtin University, GPO Box U1987, Perth, WA 6845, Australia. Tel.: +61 89266 2367; E-mail: s.egan@ curtin. edu.au.
}

and motor decline [6] and poorer quality of life and increased mortality compared to non-depressed PD patients [7,8]. The prevalence of anxiety disorders in PD varies from 25 to 45 per cent, and anxiety disorders are frequently comorbid with depression [9]. PD patients with depression and comorbid anxiety have more severe PD symptoms, show poorer response to treatment for depression, and have higher functional impairment [10].

\section{TREATMENT OF ANXIETY AND DEPRESSION IN PARKINSON'S DISEASE}

Given the evidence of poorer outcomes for PD patients with depression and anxiety, it is imperative they receive treatment. Despite this, only 10 to 20 per cent of PD patients receive treatment for depression or anxiety [11]. Pharmacotherapy is the first-line treatment. In the most recent meta-analysis that included randomized placebo controlled treatments for depression and anxiety in PD it was reported that the pooled effect of antidepressants for depression in PD was moderate $(d=0.71)$ but non-significant 
and the secondary effect of antidepressants on anxiety in PD was large $(d=1.13)$ but also non-significant [12]. Interestingly, in this meta-analysis CBT was shown to have a larger effect size $(d=1.57)$ than the antidepressant treatments [12]. As the data is still at an early stage and especially with pharmacological studies outnumbering psychological studies it is premature to compare modalities. In the meta-analysis only two single trials of non-antidepressant treatments for depression in PD were found to have significant effects, that were large, Omega-3 supplementation $(d=0.92)$ and CBT $(d=1.57)$. While there is recent literature which has highlighted some promise for other non-pharmacological interventions, such as adapted exercise programs, as yet the data is preliminary. One case study described exercise as reducing depression and improving cognitive and motor functioning [13], and another open trial with nine PD patients indicated an exercise program resulted in decreased depression and motor symptoms [14]. Despite the high prevalence of depression and anxiety in PD there is still a lack of controlled trials for both pharmacological and nonpharmacological treatments for depression and anxiety in PD which limits conclusions [12]. Given the concerns that have been raised regarding the safety and side effects of pharmacological treatments [15], it is not surprising a recent review has indicated that CBT is a promising treatment option [16].

\section{EFFICACY OF CBT FOR DEPRESSION AND ANXIETY IN PARKINSON'S DISEASE}

A range of case studies [17-20], single case experimental design series [21, 22] and open trials [23, 24], have reported CBT results in decreases in depression and anxiety in PD patients. However it is difficult to generalize from these studies with small sample sizes and lack of control groups. There have only been two randomized controlled trials (RCTs) to date examining the impact of CBT on depression in PD. In the largest study to date 80 participants with PD and depression were randomized to receive 10 sessions of individual CBT plus clinical monitoring (CM) compared to clinical monitoring only [26]. CBT participants had significantly greater reductions in HAM-D depression scores, and there were significantly more treatment responders in the $\mathrm{CBT}+\mathrm{CM}$ group compared to the CM group (56\% versus $8 \%$ ). Gains were maintained at one-month follow-up. In a recent RCT [27] of group CBT a waitlist-controlled design was used with 18 adults with PD and a diagnosis of depression and/or anxiety. In the second study, participants were randomized to either an 8 week CBT group or 8 week waitlist consisting of clinical monitoring preceding treatment. At post-treatment CBT participants reported greater reductions in depression than waitlist participants of a large effect $(d=1.12)$. Large secondary effects on anxiety were also observed for CBT participants $(d=0.89)$. Treatment gains were maintained and further improved during follow-up, where at 6-month follow-up, significant and large effects were observed in the CBT group for both depression $(d=2.07)$ and anxiety $(d=2.26)$. It is particularly noteworthy that these very large effect sizes of greater than 2.0 at 6 month follow-up indicate not only the durability of effects of group CBT on depression and anxiety, but also that treatment gains are furthered over time. These studies are encouraging given the large effect sizes found particularly in reduction of depression in $\mathrm{PD}$, however more research is still required with RCTs with adequate sample sizes and longer follow up periods.

\section{RATIONALE FOR A COGNITIVE BEHAVIORAL MODEL OF ANXIETY AND DEPRESSION IN PARKINSON'S DISEASE}

There is a need to develop a specific CBT conceptualization for depression and anxiety in PD. As yet no PD-specific CBT conceptual framework exists. Central to CBT is the concept that an individual's appraisal of their experience determines its impact. In this way two individuals may experience similar situations yet show markedly different emotional responses. Appraisal of the meaning of being diagnosed with a degenerating illness such as PD will influence ones response and coping [28]. CBT clinicians seek to understand the individual experience of the diagnosis of PD and the meanings associated with this as it may determine how the person copes with PD. The opinions and views of those around the person with PD are important as sometimes carers inadvertently reinforce a sense of dependence in the person with PD. CBT involving carers of people with PD may be empowering of both partners in the dyad as the problem-solving orientation of CBT. We suggest that partners and carers should be directly involved in treatment, where possible, and if appropriate given the nature of the relationship, for example, if it is someone that the PD patient has a relatively good relationship with. It is suggested that the partner/carer with the patient's consent, attends part of the formulation feedback session (usually session two), where the CBT therapist and patient have already 
worked out in a collaborative manner in the first half of the session an individualized model of what specific cognitive and behavioral factors are maintaining their depression and anxiety symptoms, and explain this to the partner/carer. The patient and therapist can then directly ask the partner/carer for their assistance in the treatment strategies to address these maintaining factors. For example, a partner may help behavioral experiments regarding independence by doing less for the person with PD, and a carer may help address experiments aimed at reducing isolation by facilitating the patient's attendance at social gatherings. A comprehensive conceptualization should involve conceptual level suggestions for treatment modification including taking account of individual health beliefs, beliefs about disability, cohort beliefs (which are beliefs shared amongst a peer group born in the same generation) and core beliefs [29].

\section{A COGNITIVE BEHAVIORAL MODEL}

A case example is outlined to demonstrate the benefits of developing a case conceptualization based on a guiding model of CBT for anxiety and depression in $\mathrm{PD}$ as shown in Fig. 1.

Bill was a 73 year old retired shopkeeper who presented with symptoms of generalized anxiety disorder and depression. He had watched his best friend die of end stage PD one year prior to his own diagnosis of PD. Understandably, he became anxious the same future awaited him when he received his diagnosis. Bill became depressed and stopped doing the activities that gave his life meaning and pleasure such as photography. He believed that his PD would prevent him doing photography well and thus he gave away all of his equipment as he

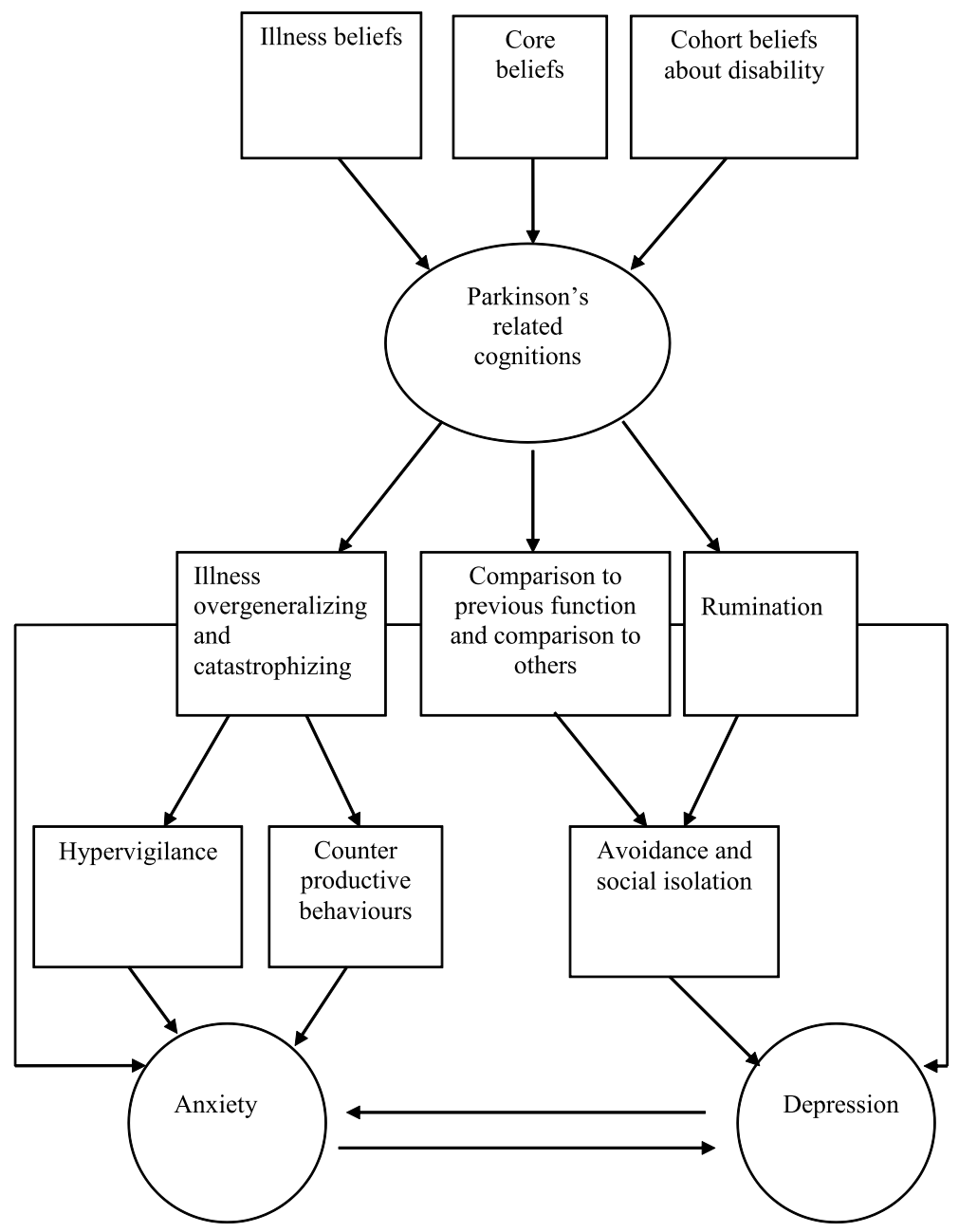

Fig. 1. A cognitive behavioral model of the development and maintenance of depression and anxiety in Parkinson's disease. 
thought "my life is over because of my PD" and "I am going to have to stop doing this sooner or later so may as well get the pain over with now". Bill also reported isolating himself due to embarrassment over his motor symptoms, for example worrying he would spill food and drinks. He stopped attending a music club with his friends and also reduced his interest in buying music to share. Bill also stated that since his wife had died five years ago he had started to go out and meet with friends, but since his diagnosis of PD had withdrawn from his friends, felt more lonely and was missing her more. He reported depressive rumination about his $P D$ and hopelessness about the future. Bill also worried about being depressed, and that he was losing his identity and was "weak". He was worried he would get to the "point of no return" with his PD and as a result avoided doing many things where his symptoms would be noticeable, and became hypervigilant about his symptoms looking for the start of the "end". Bill also reported thinking "I am incompetent and can do nothing at all" and "I am completely not able to function, my life is over".

The CBT model outlined in Fig. 1 is a new model that has not been outlined before, and is based on standard cognitive-behavioral models [30] where core beliefs are defined as rigid and inflexible beliefs which are developed due to early life experiences and then activated later in life due to stressors such as the diagnosis of PD. In the case of Bill his core belief "I am incompetent" lead him to value being able to try and prove his competence through being highly independent and solving problems and he thus had associated beliefs that "A man stands on his two feet" and "If I cannot do things by myself, then I am incompetent."

There are also illness beliefs which arise from core beliefs that make someone more likely to react with negative emotions to $\mathrm{PD}$, for Bill this was watching his best friend die of PD, and was linked with his fear "I may die at any stage due to my PD". Cohort beliefs refer to values and beliefs shared amongst a generational group of individuals born at a similar time period [31]. For a man of Bills' generation, he possessed a common cohort belief that "people who are disabled are weak, needy and dependent".

CBT for depression and anxiety disorders in PD focuses on challenging the negative cognitions that maintain distress in PD. Negative PD related cognitions are over-generalized in nature, for example in Bill's case his cognition was "Having PD is terrible it means my life is over". As a result of this negative cognition he experienced a number of specific secondary cognitive biases. These can involve illness overgeneralizing and catastrophizing, for example Bill thought "these tremors are terrible I might as well sell my photography equipment and car now I am going to lose everything eventually". Bill overgeneralized the level of impairment he is experiencing with PD as he remains currently able to function independently but is catastrophizing about reduction in future functioning.

Another negative cognition that maintains depression in PD is comparison to previous function and function of others. This involves an unhelpful process of comparing one's current functioning with functioning prior to PD, for example Bill thinks "I used to do this [task] without thinking now look what I have come to" and "everyone else can do simple things without thinking about it". This negative cognition leads to self-criticism resulting in hopelessness. Rumination also involves hopelessness regarding the future and current state of disability. Bill's rumination centered on "why have I got this, it is unfair, I am a burden" which maintained his depression. Hypervigilance refers to constantly scanning physical symptoms which may signal decline in functioning. In Bill's case this involved monitoring himself for tremors, and he interpreted the presence of tremors as evidence for his illness overgeneralizing and catastrophizing beliefs. Another behavioral factor is counterproductive behaviors (also known as safety behaviors), and these are often seen in the individual with anxiety about their PD. For example, Bill's counterproductive behavior was to tightly hold his hands when in public to avoid his resting tremor being noticeable to others. The problem however was that it made his tremors worse, further reinforcing anxiety regarding the tremors. Avoidance is a very common behavioral response and involves avoidance of situations the individual finds distressing due to their PD symptoms. Bill avoided eating in front of others due to a concern that he might spill food or drink, even though this had not happened before, which resulted in social isolation. Another factor which was important in Bill's case was his feelings of loneliness and missing his wife who had died, which interacted with his withdrawal and social isolation as a result of concern over his PD symptoms.

This model is new and has not been proposed before, although combines the commonly known important cognitive and behavioral maintaining factors of anxiety and depression including core beliefs [30], rumination [31], hypervigilance for physical symptoms [32], counterproductive (safety) behaviors [33], and avoidance [34]. While these are common factors that are 
included in general cognitive behavioral maintenance models, they can be specifically adapted to suit how they are maintaining anxiety and depression for the individual with PD as outlined in the example. In addition to these general factors which can be adapted in an individualized case formulation with the patient with PD, the model also considers several factors which are specific to PD such as illness beliefs, cohort beliefs, and comparison to previous function/function of others. Given this model is new it would be useful for future research to examine if the model has validity in accounting for the maintenance of anxiety and depression in Parkinson's disease. This could be examined in future research by determining the predictive validity of the model by including measures of the factors specified in the model, e.g., rumination, avoidance, with measures of anxiety and depression in individuals with PD to determine the degree to which the factors can account for symptoms. However this would only be an indirect test of the model, the model could be more directly tested in a large sample of PD patients through the use of structural equation modeling to determine if the pathways between the hypothesized factors and anxiety and depression are found.

\section{GUIDELINES FOR CBT FOR DEPRESSION AND ANXIETY IN PARKINSON'S DISEASE}

In terms of which patients it is appropriate to use CBT with, we suggest that this treatment is utilized in PD patients who do not have dementia. In trials we have typically excluded patients who score less than 24 on the Mini Mental State Examination. It is also helpful if the patient expresses some degree of willingness to attend psychotherapy, those who have been referred by a partner/carer but who are reluctant to attend of their own accord may be difficult to engage in therapy. In a recent book on CBT with older people, guidelines for working with clients with chronicity and comorbidity, Laidlaw [35] updated previous recommendations [36] that clinicians may find useful when working with clients presenting with depression and anxiety in PD $[35,36]$. These include;

(1) Practical barriers to participation in therapy. One of the most common reasons that patients with PD do not come to therapy or dropout is practical problems in accessing therapy such as transport to sessions, or concerns over motor symptoms in session. To overcome this in trials of CBT for depression and anxiety in PD we have typically provided vouchers for taxis for patients to attend sessions so they are not reliant on carers or others to transport them to session or needing to catch public transport if they cannot or do not wish to. However, in the reality of clinical practice if funds are not available for taxis, then it is useful to see if a carer or friend can help with transport. For example, in Bill's case although he used public transport he was concerned about getting lost coming to a new clinic so a friend provided him with transport a to reduce this barrier. Barriers to therapy can also be overcome by the use of telemedicine and videoconferencing as a means to deliver therapy in the client's home who is not able to attend the clinic or lives in a rural or remote area. Physical barriers can be overcome by ensuring that the patient with PD is offered regular breaks during sessions, and session times scheduled around what is typically a better period for them for motor symptoms during the day. We recommend that clinicians outline these issues in a phone screening session with the client prior to their first appointment to remove barriers and set the scene that it is possible to engage in therapy even with physical limitations that may be present. We also suggest that in session writing is reduced, and formats of handouts are adapted if necessary for the individual, for example with larger font in handouts and thought records.

(2) Accepting depression as a separate but reversible problem distinct from their ongoing physical problems. One of the most common presentations that are seen in PD patients with depression is confusion over the symptoms of depression and the patient attributing symptoms of depression to Parkinson's disease. Often patients are surprised when feedback is given regarding a diagnosis of depression, and that it is possible for depression to improve as they believed the way they were feeling was a part of PD. For example, Bill reported that he thought the low mood and anxiety he felt was just a part of living with PD and was surprised when the therapist suggested that they could help to remove those symptoms. This is an important point to collaboratively discuss with patients as it can help them particularly at the start of therapy to feel more hopeful about the future and more engaged in the therapeutic process if they realize that it is possible to have a significant improvement in their mood and quality of life that is independent from the process of the 
disease of PD, that they are not "stuck" with feeling depressed and anxious due to the PD that it is distinct from PD and can be improved.

(3) Identifying and limiting excess disability. It is important to identify in a collaborative manner with patients the extra disability that symptoms of depression and anxiety are adding to their life in addition to the symptoms of PD. This can be done in an initial formulation session with the patient, where the impact of symptoms of depression and anxiety on their life are explored. For example, Bill had stopped doing many pleasant events such as photography as he perceived he would no longer be able to do them due to his symptoms of PD, but when this was explored with Bill it was discovered that in fact he could still do the photography with his current motor symptoms, but was concerned that in the future it would become so bad he would have to give it up. The therapist helped Bill to understand that by reengaging in pleasant activities that he could indeed still engage in he would start to feel better, and that even if he did find in the future that an activity was more difficult due to his PD symptoms, that a more helpful approach would be to adjust how he did the event rather than give up doing it, as most activities could be engaged in with some degree of adjustment while still enjoying them.

(4) Recognizing and limiting losses of roles and autonomy. Many patients with PD report low mood associated with a loss of roles on diagnosis of PD, for example, if patients stop working, or doing other valued activities, and also frustration at a loss of autonomy, for example if they rely on others to help with daily self-care activities or for transport. It is useful to help the patient to see that while there may indeed be some loss of roles due to the PD, and to validate these losses in terms of the grief and loss regarding this, that not all roles are necessarily lost. For example, Bill still had a wide circle of friends despite having withdrawn from them and his previous roles of organizing events with friends for music and photography were still possible if he reengaged with them. Regarding loss of autonomy, in Bill's case he was distressed when he sometimes had to ask others for a lift to an appointment and so avoided doing so and often missed medical appointments or suffered inconvenience due to difficulty with public transport. The therapist helped Bill to see that it was ok to sometimes ask others for lifts as they liked to help, and while he had lost some autonomy he was still able to function independently in most aspects of his life, and that he could feel better by offering help to friends in return for their favors.

(5) Challenging self-perception of oneself as a burden to others. It is very common for patients with PD to report feeling concerned that they are a burden to carers. In Bill's case he reported feeling like a burden to his friends if he asked them for favors, therefore in a similar manner to the previous point, the therapist helped Bill to understand the positive effect of helping others on the friend feeling good for helping, and also for the social interaction that occurred as a result. Bill was encouraged by the therapist to think of other ways that he contributed to his friendships and was not a burden, for example he had helped a friend with his tax return and this was commented on by the friend as being a very helpful activity that Bill did.

CBT for PD typically involves 8-12 one hour sessions that are conducted on a weekly basis. Examples of the CBT strategies that can be employed for the maintaining factors proposed in the model are outlined in Table 1. It should be noted that therapy is not intended to be a strict session by session protocol, rather as is commonplace in most modern CBT

Table 1

Cognitive behavioral therapy strategies to address the proposed maintaining factors of anxiety and depression in Parkinson's disease

\begin{tabular}{ll}
\hline Maintaining factor & Strategy \\
\hline Avoidance and Social Isolation & Behavioral activation, thought diaries \\
Rumination & Thought diaries \\
Comparison to previous function and comparison to others & Grief therapy, thought diaries \\
Illness overgeneralizing and catastrophizing & Thought diaries, behavioral experiments \\
Counterproductive behaviors & Behavioral experiments \\
Hypervigilance & Behavioral experiments, attention refocusing \\
\hline
\end{tabular}


approaches for various problems, therapy should be tailored to the individual based on an individualized formulation of the person's problems and disease severity. The model should be used as a guide to help develop this individualized formulation, and then therapy and which specific techniques are to be used based on the particular individual formulation. For example, not all maintaining factors that are proposed in the model may be present for a particular person, and therefore only the maintaining factors that are important to the individual should be targeted, and the specific strategies that can be used to address these as shown in Table 1.

In the first phase of treatment, emphasis is placed on breaking cycles of negative thoughts about impairment due to PD. The early sessions address the first three elements outlined of practical barriers, seeing depression as separate but reversible condition and decreasing excess disability through starting behavioral experiments to challenge beliefs regarding disability. Clinicians should also establish goals for treatment and engage in psychoeducation regarding anxiety disorders and depression in this early phase. Psychoeducation generally occurs around session 2-3 and involves the patient understanding what are symptoms of anxiety and the fight or flight response, what symptoms constitute depression and how these are maintained for example by negative cognitions, social withdrawal and lack of engagement in pleasant events. Typically psychoeducation can be conducted in the same session as the collaborative formulation where the CBT therapist is addressing with the patient the understanding of why their problem has arisen and what factors are maintaining their depression and anxiety, and can be continued in the next session if required. In the initial phase of treatment the clinician should also engage caregivers in treatment, given caregiver participation, in particular spouses, has been found to positively influence treatment response to CBT for depression in PD [37].

In the mid-phase of treatment, clinicians challenge beliefs regarding limiting losses of roles and autonomy by introducing the experience of diagnosis as being like a grief process. A phase model of grief [38] (i.e. Worden defined stages of mourning; bargaining and denial, anger, acceptance and moving on) can help clients come to terms with PD and focus more explicitly upon what aspects of life they appraise to be most keenly felt in terms of loss post-diagnosis. While becoming aware of one's limitations can be painful, it can also promote a realistic appraisal and hence adjustment to living with PD. This is captured in the framework for optimizing function in the face of realistic challenges of ageing. Baltes and colleagues developed Selective, Optimization with compensation to explain how individuals can proactively and intentionally make adjustments in order to optimize functioning in light of age-related challenges to wellbeing [39]. This recognizes goals can be attained through alternative means and assists the client to function at their most optimal level.

Although people living with PD often have to deal with realistic challenges, individuals nevertheless often endorse unhelpful or erroneous cognitions about these challenges. Challenging these unhelpful cognitions is the primary focus of the middle phase of therapy. Behavioral experiments are used extensively in this phase. A behavioral experiment challenges negative cognitions by testing out a specific negative prediction an individual believes will occur [40] and is a highly effective technique [41]. Bill tested out his belief that he would be incompetent if he made a mistake by testing his negative prediction that others would laugh at him if he spilled his food and drink. He first tested out in session if he would spill food or drink and then out for lunch with his friends. The result of the experiment was that Bill spilled a small amount of drink but not an entire glass as he had predicted and his friends did not notice or say anything. As a result, he concluded others are unlikely to laugh at him in public and this helped him to go out and decrease his social isolation which resulted in decreased depressive symptoms. Cognitive restructuring focuses on challenging unhelpful thoughts about the impact of PD perceived limitations due to PD. Thought diaries are useful in challenging negative PD related specific cognitions and cognitive biases. Thought diaries are used to challenge any negative cognition the individual with PD is experiencing as it is these negative cognitions which are seen as maintaining depression and anxiety disorders in PD. Behavioral activation which has extensive evidence of efficacy in the treatment of depression in older adults [42] involves engaging the person is pleasant and rewarding events is also used to improve mood, along with decreasing avoidance and social isolation. Furthermore, behavioral activation has been shown to be effective in reducing depression when delivered to older adults via videoconferencing [42] so is highly suitable for a PD population where barriers to accessing therapy in a clinic are a problem.

The final stage of CBT involves individualized relapse prevention to ensure $\mathrm{CBT}$ is continued. The aim of CBT is for the person to become their own therapist and to continue to use the cognitive techniques of thought diaries, behavioral experiments, and 
behavioral activation to challenge negative cognitions and symptoms of depression and anxiety in the future.

\section{SUMMARY}

There is an important need to develop CBT for anxiety and depression in PD. It is hoped that this model of CBT helps clinicians to further research on efficacy and lead to widespread dissemination of CBT as a viable treatment option for depression and anxiety in $\mathrm{PD}$ given the promising evidence of efficacy $[3,12]$.

\section{DISCLOSURE STATEMENT}

The authors have no actual or potential conflicts of interest.

\section{REFERENCES}

[1] Dorsey ER, Constantinescu R, Thompson JP, Biglan KM, Holloway RG, Kieburtz K, Marshall FJ, Ravina BM, Schifitto G, Siderowf A, \& Tanner CM (2007) Projected number of people with Parkinson disease in the most populous nations, 2005 through 2030. Neurology, 68, 384-386.

[2] Hughes TA, Ross HF, Mindham RH, \& Spokes EG (2004) Mortality in Parkinson's disease and its association with dementia and depression. Acta Neurol Scand, 110, 118-123.

[3] Pachana NA, Egan SJ, Laidlaw K, Dissanayaka N, Byrne GJ, Brockman S, Marsh R, \& Starkstein S (2013) Clinical issues to consider in the treatment of anxiety and depression in older adults with Parkinson's disease. Mov Disord, 28, 1930-1934.

[4] Zesiewicz TA, Gold M, Chari G, \& Hauser RA (1999) Current issues in depression in Parkinson's disease. Am J Geriatr Psychiatry, 7, 110-118.

[5] Veazey C, Aki S, Cook KF, Lai EC, \& Kunik ME (2005) Prevalence and treatment of depression in Parkinson's disease. J Neuropsychiatry Clin Neurosci, 17, 310-323.

[6] Starkstein SE, Mayberg HS, Leiguarda R, Preziosi TJ, \& Robinson RG (1992) A prospective longitudinal study of depression, cognitive decline, and physical impairments in patients with Parkinson's disease. J Neurol Neurosurg Psychiatry, 55, 377-382.

[7] Dobkin RD, Allen LA, \& Menza M (2007) Cognitivebehavioural therapy for depression in Parkinson's disease: A pilot study. Mov Disord, 7, 946-952.

[8] Hughes TA, Ross HF, Mindham RH, \& Spokes EG (2004) Mortality in Parkinson's disease and its association with dementia and depression. Acta Neurol Scand, 110, 118-123.

[9] Leentjens AFG, Dujardin K, Marsh L, Martinez-Martin P, Richard IH, \& Starkstein SE (2008) Anxiety rating scales in Parkinson's disease: Critique and recommendations. Mov Disord, 23, 2015-2025.

[10] Dissanayaka NNW, Sellbach A, Silburn PA, O'Sullivan JD, Marsh R, \& Mellick GD (2011) Factors associated with depression in Parkinson's disease. J Affect Disord, 132, 82-88.

[11] Frisina PG, Borod JC, Foldi NS, \& Tenenbaum HR (2008) Depression in Parkinson's disease: Health risks, etiology, and treatment options. Neuropsychiatr Dis Treat, 4, 81-91.
[12] Troueng L, Egan SJ, \& Gasson N (2013) A meta-analysis of randomised placebo-controlled treatment trials for depression and anxiety in Parkinson's disease. PloS One, 8, e79510.2013.

[13] Lattari E, Pereira-Junior PP, Neto GA, Lamego MK, Moura AM, de Sá AS, Rimes RR, Manochio JP, Arias-Carrión O, Mura G, Nardi AE, \& Machado S (2014) Effects of chronic exercise on severity of quality of life and functionality in an elderly Parkinson's disease patient: Case report. Clin Pract Epidemiol Ment Health, 10, 126-128.

[14] Cugusi L, Solla P, Zedda F, Loi M, Serpe R, Cannas A, Marrosu F, \& Mercuro G (2014) Effects of an adapted physical activity program on motor and non-motor functions and quality of life in patients with Parkinson's disease. Neurorehabilitation, 35, 789-794.

[15] Burns DJ (2002) Beyond the iron mask: Towards better recognition and treatment of depression associated with Parkinson's disease. Mov Disord, 17, 445-454.

[16] Charidimou A, Seamons J, Selai C, \& Schrag A (2011) The role of cognitive-behavioural therapy for patients with depression in Parkinson's Disease. Parkinsons Dis, 2011, 737523.

[17] Gupta R (2000) Treatment of depression in an elderly Asian Indian male: A cognitive behavioural approach. Clin Gerontolt, 22, 87-89.

[18] Mohlman J, Reel DH, Chazin D, Ong D, Georgescu B, Tiu J, \& Dobkin RD (2010). A novel approach to treating anxiety and enhancing executive skills in an older adult with Parkinson's Disease. Clin Case Stud, 9, 74-90.

[19] Ellgring H, Seiler S, Perleth B, Frings W, Gasser T, \& Oertel W (1993) Psychosocial aspects of Parkinson's disease. Neurology, 43, 41-44.

[20] Dreisig H, Beckmann J, Wermuth L, Skovlund S, \& Bech P (1999) Psychologic effects of structured cognitive psychotherapy in young patients with Parkinson's disease: A pilot study. Nord J Psychiatry, 53, 217-221.

[21] Cole K, \& Vaughan FL (2005) Brief cognitive behavioural therapy for depression associated with Parkinson's disease: A single case series. Behav Cogn Psychotherapy, 33, 89-102.

[22] Feeney F, Egan SJ, \& Gasson N (2005) Treatment of depression and anxiety in Parkinson's disease: A pilot study using group cognitive behavioural therapy. Clin Psychologist, 9, 31-38.

[23] Farabaugh A, Locascio JJ, Yap L, Growdon J, Fava M, Crawford C, Matthews J, McCutchen J, Buchin J, Pava J, \& Alpert JE (2010) Cognitive-behavioural therapy for patients with Parkinson's disease and comborbid major depressive disorder. Psychosomatics, 51, 124-129.

[24] Dobkin RD, Allen LA, \& Menza M (2007) Cognitivebehavioral therapy for depression in Parkinson's disease: A pilot study. Mov Disord, 22, 946-952.

[25] Hamilton M (1960) A rating scale for depression. J Neurol Neurosurg Psychiatry, 23, 56-62.

[26] Dobkin RD, Menza M, Allen LA, Gara MA, Mark MH, Tiu J, Bienfait KL, \& Friedman J (2011) Cognitive-behavioral therapy for depression in Parkinson's disease: A randomized, controlled trial. Am J Psychiatr, 168, 1066-1074.

[27] Troeung L, Egan SJ, \& Gasson N (2014) A randomized controlled trial of group cognitive behavioral therapy for depression and anxiety in Parkinson's disease. BMC Psychiatr, 14, 19.

[28] Dobkin RD, Menza M, \& Bienfait KL (2008) CBT for the treatment of depression in Parkinson's disease: A promising non-pharmacological approach. Expert Rev Neurother, 8 , 27-35. 
[29] Laidlaw K, \& McAlpine S (2008) Cognitive behaviour therapy: How is it different with older people? J Ration Emot Cogn Behav Ther, 26, 250-262.

[30] Beck JS (2011) Cognitive behaviour therapy: Basics and beyond, 2nd edition, Guilford, New York.

[31] Ehring T, \& Watkins ER (2008) Repetitive negative thinking as a transdiagnostic process. Int J Cogn Ther, 1, 192-205.

[32] Clark DM (1986) A cognitive approach to panic. Behav Res Ther, 24, 461-470.

[33] Salkovskis PM, Clark DM, Hackmann A, Wells A, \& Gelder MG (1999) An experimental investigation of the role of safety-seeking behaviours in the maintenance of panic disorder with agoraphobia. Behav Res Ther, 37, 559-574.

[34] Barlow DH, Farchione TJ, Fairholme CP, Ellard KK, Boisseau CL, Allen LB, \& Ehrenreich-May J (2010) The Unified Protocol for Transdiagnostic Treatment of Emotional Disorders: Therapist Guide, Oxford University Press, New York.

[35] Laidlaw K (2015) Introducing Cognitive Behaviour Therapy for Older People, SAGE, London.

[36] Rybarczyck B, Gallagher-Thompson D, Rodman J, Zeiss A, Gantz FE, \& Yesavage J (1992) Applying cognitivebehavioral psychotherapy to the chronically ill elderly: Treatment issues and case illustration. Int Psychogeriatr, 4, $127-140$.
[37] Dobkin RD, Rubino JT, Allen LA, Friedman J, Gara MA, Mark MH, \& Menza M (2012) Predictors of treatment response to cognitive-behavioral therapy for depression in Parkinson's disease. J Consult Clin Psychol, 80, 694-699.

[38] Worden JW (2009) Grief Counselling and Grief Therapy: A Handbook for the Mental Health Practitioner, fourth edition, Springer, New York.

[39] Freund AM, \& Baltes PB (1998) Selection, optimization, and compensation as strategies of life management: Correlations with subjective indicators of successful aging. Psychol Aging, 13, 531-543.

[40] Bennett-Levy J, Butler G, Fennell M, Hackmann A, Mueller M, \& Westbrook D (2004) The Oxford Guide to Behavioral Experiments in Cognitive Therapy, Oxford University Press, New York.

[41] McManus F, van Doorn K, \& Yiend J (2012) Examining the effects of thought records and behavioral experiments in instigating belief change. $J$ Behav Therapy Exp Psychiatry, 43, 540-547.

[42] Lazzari C, Egan SJ, \& Rees CS (2011) Behavioral activation treatment for depression in older adults delivered via videoconferencing. Cogn Behav Pract, 18, 555-565. 